PROCEEDINGS OF THE

AMERICAN MATHEMATICAL SOCIETY

Volume 125, Number 12, December 1997, Pages 3669-3673

S 0002-9939(97)04207-X

\title{
WAVELET BASES IN REARRANGEMENT INVARIANT FUNCTION SPACES
}

\author{
PAOLO M. SOARDI
}

(Communicated by J. Marshall Ash)

\begin{abstract}
We point out that the well known characterization of $L^{p}$ spaces $(1<p<\infty)$ in terms of orthogonal wavelet bases extends to any separable rearrangement invariant Banach function space $X$ on $R^{n}$ (equipped with Lebesgue measure) with nontrivial Boyd's indices. Moreover we show that such bases are unconditional bases of $X$.
\end{abstract}

\section{WAVELETS IN R.I. FUNCTION SPACES}

The purpose of this note is to observe that the well known characterization of $L^{p}\left(\mathbb{R}^{n}\right), 1<p<\infty$, in terms of orthonormal wavelet bases [M, Theorem VI. 1] extends to any separable rearrangement invariant (r.i.) Banach function space $X$ on $\mathbb{R}^{n}$ (equipped with Lebesgue measure) with nontrivial Boyd's indices. Moreover we show that such bases are unconditional bases of $X$.

The interest in Boyd's indices originated from Boyd's interpolation theorem: every quasilinear operator of joint weak type $(p, p ; q, q)$ is bounded from $X$ to $X$ if and only if the Boyd indices $\underline{\alpha}$ and $\bar{\alpha}$ of $X$ satisfy $q^{-1}<\underline{\alpha} \leq \bar{\alpha}<p^{-1}$. The reader is referred to $[\mathrm{B}-\mathrm{S}]$ for the relevant definitions and properties of Banach function spaces and interpolation theory.

Even though the extension mentioned above follows from a straightforward application of Boyd's theorem, we think it worthwhile to point out this characterization of a remarkable class of spaces. In fact this class includes the Lorentz spaces $L^{p, q}\left(\mathbb{R}^{n}\right)$ with $1<p<\infty, 1 \leq q<\infty$, and all the reflexive Orlicz function spaces $L^{\Phi}\left(\mathbb{R}^{n}\right)$.

From now on let $\psi_{j, k, \ell}(x)=2^{j n / 2} \psi_{\ell}\left(2^{j} x-k\right.$ ) (where $j \in \mathbb{Z}, k \in \mathbb{Z}^{n}$ and $\ell=$ $1,2, \ldots, 2^{n}-1$ ) be an orthonormal wavelet basis associated with a $r$-regular (in the sense of Meyer) multiresolution analysis of $L^{2}\left(\mathbb{R}^{n}\right)$. We shall adopt the shorter notation $\psi_{j, k, \ell}=\psi_{\lambda}, \lambda \in \Lambda$. For every $\lambda=(j, k, \ell)$ we will also denote by $Q(\lambda)$ the dyadic cube $\left\{x: 2^{j} x-k \in\left[0,1\left[^{n}\right\}\right.\right.$ and by $\chi_{\lambda}$ its characteristic function.

For every function $f \in L^{1}+L^{\infty}$ we may define the coefficients

$$
c(\lambda)=\int_{\mathbb{R}^{n}} f(x) \bar{\psi}_{\lambda}(x) d x .
$$

Before stating our main result, let us point out two important properties of separable Banach function spaces, which will be used in the proof of Proposition 1.

Received by the editors July 17, 1996.

1991 Mathematics Subject Classification. Primary 42C15.

(C)1997 American Mathematical Society 
First, by [B-S, Corollary 5.6, p. 29] every separable Banach function space on $R^{n}$ equipped with Lebesgue measure has absolutely continuous norm; i.e., for every $f \in X$ and for every sequence of measurable sets $E_{n}$ such that $E_{n} \rightarrow \emptyset$ a.e. we have that $\left\|f \chi_{E_{n}}\right\|_{X} \rightarrow 0$. Combining this property with [B-S, Theorem 3.13, p. 19] we obtain that a separable Banach function space is always the closure of the subspace of the bounded functions supported in sets of finite measure .

Proposition 1. Let $X$ be a separable rearrangement invariant Banach function space on $\mathbb{R}^{n}$ equipped with Lebesgue measure and let $\left\{\psi_{\lambda}\right\}_{\lambda \in \Lambda}$ be an orthonormal wavelet basis associated with a r-regular multiresolution analysis of $L^{2}\left(\mathbb{R}^{n}\right)$.

Let $\underline{\alpha}$ and $\bar{\alpha}$ denote the Boyd indices of $X$. If $0<\underline{\alpha} \leq \bar{\alpha}<1$, then $\left\{\psi_{\lambda}\right\}_{\lambda \in \Lambda}$ is an unconditional basis of $X$ and the three norms

$$
\|f\|_{X}, \quad\left\|\left(\sum_{\lambda}\left|c(\lambda) \psi_{\lambda}\right|^{2}\right)^{1 / 2}\right\|_{X}, \quad\left\|\left(\sum_{\lambda}|c(\lambda)|^{2}|Q(\lambda)|^{-1} \chi_{\lambda}\right)^{1 / 2}\right\|_{X}
$$

are equivalent.

Proof. Let $0<1 / p_{2}<\underline{\alpha}$ and $\bar{\alpha}<1 / p_{1}<1$. Denote by $\sigma$ the sublinear operator

$$
\sigma(f)=\left(\sum_{\lambda}\left|c(\lambda) \psi_{\lambda}\right|^{2}\right)^{1 / 2}
$$

By the $L^{p}$ result, $\sigma$ is of strong type $\left(p_{i}, p_{i}\right)(i=1,2)$ and therefore $\sigma$ is of joint weak type $\left(p_{1}, p_{1} ; p_{2}, p_{2}\right)$. By Boyd's interpolation theorem $\sigma$ is a bounded operator from $X$ to itself. Hence, for some constant $C>0$

$$
\|\sigma(f)\|_{X} \leq C\|f\|_{X} \quad \text { for all } f \in X .
$$

The same argument applies to the operator

$$
\tau(f)=\left(\sum_{\lambda}|c(\lambda)|^{2}|Q(\lambda)|^{-1} \chi_{\lambda}\right)^{1 / 2},
$$

giving, for some constant $B>0$,

$$
\|\tau(f)\|_{X} \leq B\|f\|_{X} \quad \text { for all } f \in X .
$$

Conversely, let $X^{\prime}$ denote the associate Banach function space of $X$. Then $X^{\prime}$ is a r.i. Banach function space whose Boyd indices are $\underline{\alpha}^{\prime}=1-\bar{\alpha}$ and $\bar{\alpha}^{\prime}=1-\underline{\alpha}$, so that (3) holds with $X^{\prime}$ in place of $X$.

Let $f$ and $g$ be bounded functions supported in sets of finite measure. Then $f$ and $g$ belong to $L^{2}\left(\mathbb{R}^{n}\right)$, so that they have the expansion

$$
f=\sum_{\lambda} c(\lambda) \psi_{\lambda}, \quad g=\sum_{\lambda} b(\lambda) \psi_{\lambda} .
$$

By the orthonormality of the system $\left\{\psi_{\lambda}\right\}$ we get

$$
\int_{\mathbb{R}^{n}} f \bar{g} d x=\int_{\mathbb{R}^{n}} \sum_{\lambda} c(\lambda) \psi_{\lambda} \overline{b(\lambda) \psi_{\lambda}} d x .
$$

It follows that

$$
\begin{aligned}
\left|\int_{\mathbb{R}^{n}} f \bar{g} d x\right| & \leq \int_{\mathbb{R}^{n}}\left(\sum_{\lambda}\left|c(\lambda) \psi_{\lambda}\right|^{2}\right)^{1 / 2}\left(\sum_{\lambda}\left|b(\lambda) \psi_{\lambda}\right|^{2}\right)^{1 / 2} d x \\
& \leq\|\sigma(f)\|_{X}\|\sigma(g)\|_{X^{\prime}} .
\end{aligned}
$$


By (3) applied to $X^{\prime},\|\sigma(g)\|_{X^{\prime}} \leq C^{\prime}\|g\|_{X^{\prime}}$ for some constant $C^{\prime}>0$ so that

$$
\left|\int_{\mathbb{R}^{n}} f \bar{g} d x\right| \leq C^{\prime}\|\sigma(f)\|_{X}\|g\|_{X^{\prime}}
$$

Since $X^{\prime \prime}=X$ by the Lorentz-Luxemburg theorem, we may compute the norm of $f$ as

$$
\|f\|_{X}=\sup \frac{\left|\int_{\mathbb{R}^{n}} f \bar{g} d x\right|}{\|g\|_{X^{\prime}}}
$$

where the supremum is over all the bounded $g$ supported in sets of finite measure; see [B-S, Theorem 3.12, p.18]. Thus we get from (6) and (7) the reverse inequality

$$
\|f\|_{X} \leq C^{\prime}\|\sigma(f)\|_{X}
$$

for some constant $C^{\prime}>0$. By density and by the boundedness of $\sigma$ on $X$, (8) holds for all $f \in X$.

Next we prove the converse of inequality (4). Let $U$ be the unitary operator on $L^{2}\left(\mathbb{R}^{n}\right)$ such that $U\left(\psi_{\lambda}\right)=h_{\lambda}$, where $h_{\lambda}$ is the $n$-dimensional $\lambda$-th Haar function. As before, let $f$ and $g$ be bounded with support of finite measure so that $f$ and $g$ have the expansion (5). Then,

$$
\begin{gathered}
U(f)=\sum_{\lambda \in \Lambda} c(\lambda) h_{\lambda}, \quad U(g)=\sum_{\lambda \in \Lambda} b(\lambda) h_{\lambda}, \\
\tau(f)=\left(\sum_{\lambda}\left|c(\lambda) h_{\lambda}\right|^{2}\right)^{1 / 2}, \quad \tau(g)=\left(\sum_{\lambda}\left|b(\lambda) h_{\lambda}\right|^{2}\right)^{1 / 2} .
\end{gathered}
$$

By the orthonormality of the Haar system we have

$$
\int_{\mathbb{R}^{n}} f \bar{g} d x=\int_{\mathbb{R}^{n}} U(f) \overline{U(g)} d x=\int_{\mathbb{R}^{n}} \sum_{\lambda} c(\lambda) h_{\lambda} \overline{b(\lambda) h_{\lambda}} d x
$$

Hence, arguing as above, we get from (4) applied to $X^{\prime}$

$$
\left|\int_{\mathbb{R}^{n}} f \bar{g} d x\right| \leq \int_{\mathbb{R}^{n}} \tau(f) \tau(g) d x \leq B^{\prime}|| \tau(f)\left\|_{X}\right\| g \|_{X^{\prime}}
$$

for some constant $B^{\prime}>0$. Using (7) we get the reverse inequality

$$
\|f\|_{X} \leq B^{\prime}\|\tau(f)\|_{X}
$$

which can be extended to every $f \in X$.

Finally we prove that $\left\{\psi_{\lambda}\right\}_{\lambda \in \Lambda}$ is an unconditional basis for $X$. Let $\left\{\Lambda_{n}\right\}$ be an increasing sequence of finite sets such that $\Lambda_{n} \uparrow \Lambda$. Let $f \in X$ and let $c(\lambda)$ be as in (1). Since $\left(\sum_{\lambda \in \Lambda}\left|c(\lambda) \psi_{\lambda}\right|^{2}\right)^{1 / 2}$ belongs to $X$, the series converges a.e. so that

$$
\sum_{\lambda \in \Lambda \backslash \Lambda_{n}}\left|c(\lambda) \psi_{\lambda}\right|^{2} \rightarrow 0 \quad \text { a.e. as } n \rightarrow \infty .
$$

Then (8) implies

$$
\left\|f-\sum_{\lambda \in \Lambda_{n}} c(\lambda) \psi_{\lambda}\right\|_{X} \leq C^{\prime}\left\|\left(\sum_{\lambda \in \Lambda \backslash \Lambda_{n}}\left|c(\lambda) \psi_{\lambda}\right|^{2}\right)^{1 / 2}\right\|_{X} .
$$

and the last norm tends to 0 as $n \rightarrow \infty$ by the dominated convergence theorem for Banach function spaces with absolutely continuous norm.

Remark. In $[\mathrm{H}-\mathrm{W}]$ the spaces $L^{p}(\mathbb{R})$ (among other spaces) are characterized by means of more general wavelets than those considered in Proposition 1. 


\section{LORENTZ AND ORLICZ SPACES}

Proposition 1 applies to the Lorentz spaces $L^{p, q}\left(\mathbb{R}^{n}\right)$ with $1<p<\infty, 1 \leq q<\infty$. In this case both Boyd's indices are equal to 1/p; see e.g. [B-S, Theorem 4.6, p.219]. Note that the space $L^{p, 1}$ is not reflexive.

Another remarkable example is provided by Orlicz spaces. We use the definition of Orlicz spaces given in $[\mathrm{R}-\mathrm{R}]$. Let $\phi:[0,+\infty) \mapsto[0,+\infty]$ be a left-continuous, nondecreasing function such that $\phi(0)=0$ and let $\psi$ be its left-continuous inverse. Then the functions $\Phi$ and $\Psi$, defined for $x \in \mathbb{R}^{+}$by

$$
\Phi(x)=\int_{0}^{x} \phi(t) d t, \quad \Psi(x)=\int_{0}^{x} \psi(t) d t,
$$

are called complementary Young's functions.

For every measurable $f$ on $\mathbb{R}^{n}$ define the Minkowski functional as

$$
M_{\Phi}(f)=\int_{\mathbb{R}^{n}} \Phi(|f(x)|) d x .
$$

The Orlicz space $L^{\Phi}\left(\mathbb{R}^{n}\right)$ is the Banach space of all $f$ such that the LuxemburgWeiss norm is finite; i.e.,

$$
\|f\|_{L^{\Phi}}=\inf \left\{t>0: M_{\Phi}(f / t) \leq 1\right\}<\infty .
$$

It turns out that $L^{\Phi}\left(\mathbb{R}^{n}\right)$ is a r.i. function space whose associated space is $L^{\Psi}\left(\mathbb{R}^{n}\right)$ equipped with the Orlicz norm

$$
\|g\|_{\Psi}=\sup _{M_{\Phi}(f) \leq 1}\left|\int_{\mathbb{R}^{n}} f(x) g(x) d x\right| .
$$

The Orlicz and the Luxemburg-Weiss norm are equivalent on $L^{\Psi}$.

The Young function $\Phi$ is said to be $\Delta_{2}-$ regular [R-R, p.22 and p.46] if there is a constant $K$ such that

$$
\Phi(2 x) \leq K \Phi(x) \quad \text { for all } x>0 .
$$

It is known (see e.g. Proposition 2, p.112 in $[\mathrm{R}-\mathrm{R}]$ ) that $L^{\Phi}\left(\mathbb{R}^{n}\right)$ is separable if and only if $\Phi$ is $\Delta_{2}$-regular, and it is reflexive if and only if both $\Phi$ and $\Psi$ are $\Delta_{2}$-regular.

The following result is an immediate consequence of Proposition 1 and of results of Boyd.

Proposition 2. Suppose that $X=L^{\Phi}\left(\mathbb{R}^{n}\right)$ is a reflexive Orlicz space. Let $\left\{\psi_{\lambda}\right\}_{\lambda \in \Lambda}$ be an orthonormal wavelet basis associated with a $r$-regular multiresolution analysis of $L^{2}\left(\mathbb{R}^{n}\right)$. Then $\left\{\psi_{\lambda}\right\}_{\lambda \in \Lambda}$ is an unconditional basis of $L^{\Phi}\left(\mathbb{R}^{n}\right)$ and the three norms in (2) are equivalent.

Proof. It was proved by Boyd (see Lemma 3.5, Lemma 3.6 and Lemma 5.9 in [B]) that $\underline{\alpha}>0$ if and only if $\Phi$ is $\Delta_{2}$-regular. Since the complementary Young function $\Psi$ is $\Delta_{2}-$ regular as well, we may conclude that $1-\bar{\alpha}=\underline{\alpha}^{\prime}>0$.

\section{REFERENCES}

[B-S] Bennet C. and Sharpley R., Interpolation of operators, Academic Press, Boston, San Diego, New York, 1988. MR 89e:46001

[B] Boyd D. W., The Hilbert transform on rearrangement invariant spaces, Canad. J. Math. 19 (1967), 599-616. MR 35:3383 
[H-W] Hernandez E. and Weiss G., A first course on wavelets, CRC Press, Boca Raton, Ann Arbor, London, Tokyo, 1996.

[M] Meyer Y., Ondelettes et Operateurs I. Ondelettes, Hermann, 1990. MR 93i:42002

[R-R] Rao M.M and Ren Z.D., Theory of Orlicz spaces, Marcel Dekker, 1991. MR 92e:46049

Dipartimento di Matematica dell' Università, Via Saldini 50, 20133 Milano, Italy

E-mail address: soardi@vmimat.mat.unimi.it 